\title{
Kinematic Model of Anthropomorphic Robotics Finger Mechanisms
}

\author{
Abdul Haseeb Zaidy ${ }^{1}$, Wasim Akram ${ }^{2}$, P. Tauseef Ahmad ${ }^{3}$, Mohd. Parvez ${ }^{4}$ \\ ${ }^{1}$ (Research Scholar, Mechanical Engg. Department,AFSET Dhauj Faridabad, India \\ ${ }^{2}$ (Research Scholar, Mechanical Engg. Department,AFSET Dhauj Faridabad, India \\ ${ }^{3}$ (Research Scholar, Mechanical Engg. Department,AFSET Dhauj Faridabad, India \\ ${ }^{4}$ (Research Scholar, Mechanical Engg. Department,AFSET Dhauj Faridabad, India
}

\begin{abstract}
Research on Kinematic Model of Anthropomorphic robotics Finger mechanisms is being carried out to accommodate a variety of tasks such as grasping and manipulation of objects in the field of industrial applications, service robots, and rehabilitation robots. The first step in realizing a fully functional of anthropomorphic robotics Finger mechanisms is kinematic modeling. In this paper, a Kinematic Model of Anthropomorphic robotics Finger mechanism is proposed based on the biological equivalent of human hand where each links interconnect at the metacarpophalangeal (MCP), proximal interphalangeal (PIP) and distal interphalangeal (DIP) joints respectively. The Kinematic modeling was carried out using Denavit Hartenburg (DH) algorithm for the proposed of Kinematic Model of Anthropomorphic robotics Finger mechanisms.

Index Terms-Anthropomorphic robot Finger, Modeling, Robotics, Simulation
\end{abstract}

\section{Introduction}

Among the vast applications of robotics, robotic assistance in human daily life and has been the major factor that contributes to its development. The focus on the anthropomorphism robotic limbs is currently undergoing a very rapid development. The creation of a multifingered anthropomorphic robotic hand is a challenge that demands innovative integration of mechanical, electronics, control and embedded software designs.

\section{Literature Review -}

The normal human hand has a set of hand which includes palm and fingers. There are five fingers in each hand, where each finger has three different phalanxes: Proximal, Middle and Distal Phalanxes. These three phalanxes are separated by two joints, called the Interphalangeal joints (IP joints). The IP joints function like hinges for bending and straightening the fingers and the thumb. The IP joint closest to the palm is called the Metacarpals joint (MCP). Next to the MCP joint is the Proximal IP joint (PIP) which is in between the Proximal and Middle Phalanx of a finger. The joint at the end of the finger is called the Distal IP joint (DIP). Both PIP and DIP joints have one Degree of Freedom (DOF) owing to rotational movement [7]. The thumb is a complex physical structure among the fingers and only has one IP joint between the two thumb phalanxes. Except for the thumb, the other four fingers (index, middle, ring and pinky) have similar structures in terms of kinematics and dynamics features. Average range of motion among the four fingers for flexion-extension movement is 650 at the DIP joint, 1000 at the PIP joint and 800 at the MCP joint while the abduction-adduction angles for the index finger has been measured as 200 at the MCP joint[7,8]. Figure 1 illustrates the structure of a human finger.

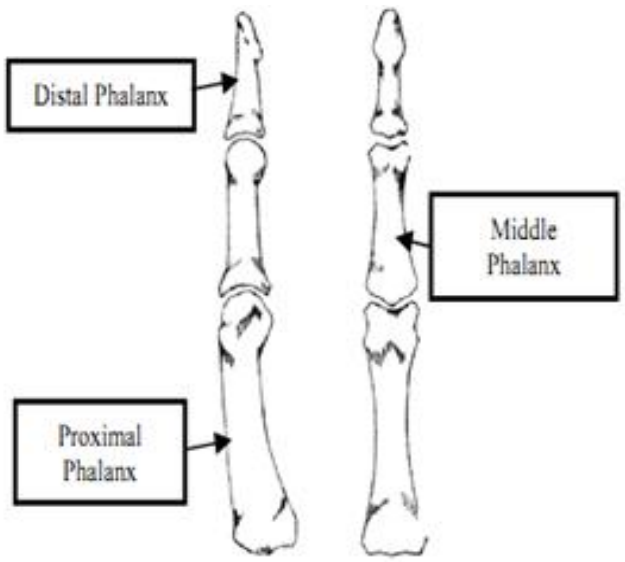

Fig.1: Structure of Human Finger 


\section{KINEMATIC RELATIONSHIP BETWEEN ADJACENT LINKS}

In 1955, Denavit and Hartenberg[1] published a paper in the ASME journal of applied mechanics that was later used to represent and model space mechanism and to derive their eq. o motion. The DH model of representation is a very simple way of modeling space mechanism links and joints that can be used for any space mechanism configuration, regardless of its sequence or complexity. It can also be used to represent transformation in any coordinates such as Cartesian, cylindrical, spherical, Euler and RPY.[2,3,4,5,6]

Kinematic modeling of space mechanism describe by D-H Notation. There are four D-H parameterstwo link parameters (ai , ai) and two joint parameters (di, $\theta \mathrm{i})$ - are defined as:

(a) Link Length (ai) -Distance measured along xi-axis from the point of intersection of xi-axis with zi-1 axis to the origin of frame $\{i\}$, that is, distance $C D$.

(b) Link twist ( $\alpha$ i) - angle between zi-1 and zi axes measured about xi axes in the right hand sense.

(c) Joint distance (di)- distance measured along zi-1 from the origin of frame $\{\mathrm{i}-1\}$ (point B) to the intersection of xi axis with zi-1 axis (point $\mathrm{C}$ ) that is, distance $\mathrm{BC}$

(d) Joint angle ( $\theta i$ )- angle between xi-1 and xi axes measured about the zi-1 axis in the right hand sense.

This notation is presented in this section and followed throughout the text Denavit and Hartenberg Representation.

First, we need to define a representation of position and orientation (pose) of one body relative to another, in the form of our transformation matrix. There are any number of general ways to do this, and several accepted conventions for doing this. In serial robotics, the Denavit and Hartenberg representation is the standard for position kinematics. In the Denavit and Hartenberg (D\&H) convention, a general transformation between two bodies is defined as the product of four basic transformations: a rotation about the initial $\mathrm{z}$ axis by $q$, a translation along the initial $\mathrm{z}$ axis by $d$, a translation along the new $\mathrm{x}$ axis by $a$, and a rotation about the new $\mathrm{x}$ axis by $a$.

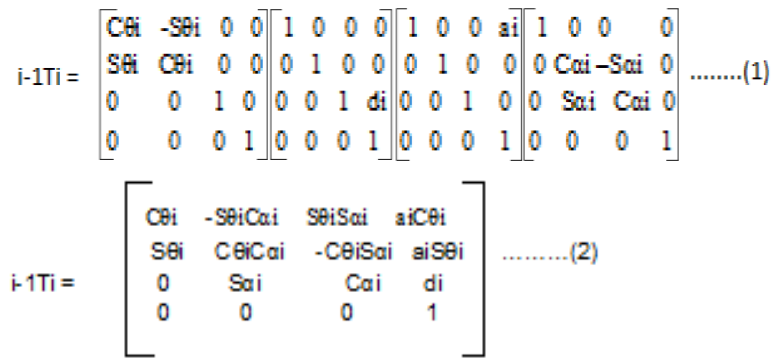

Notice now that we are able to define the general pose of one body with respect to another with only 4 parameters, q, $d, a$, and a instead of 6 which we would expect.[7] This is due to a careful choice for attaching these frames in the D-H convention and shown here.

Consider two general space mechanism links connected with joints as shown fig. 2 here.

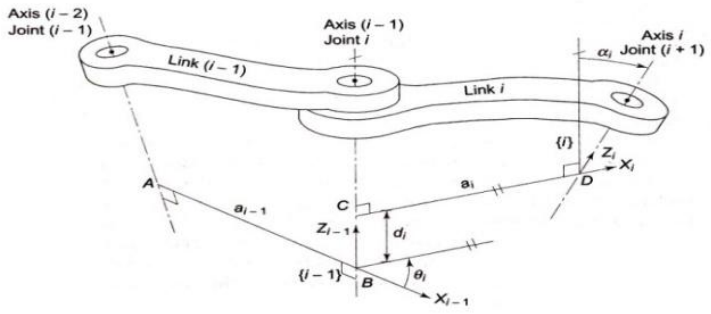

Fig.2: Joint-Link Parameters

\section{Matlab Programming}

In order to obtain eq. (2), the symbolic operations have to be performed in MATLAB. For that the following commands are used;

$>$ syms bi thi8 ai ali;

$>$ tbm $=[1,0,0,0 ; 0,1,0,0 ; 0,1, \mathrm{bi} ; 0,0,0,1]$;

$>$ tthm $=[\cos ($ thi $0,-\sin ($ thi $), 0,0 ; \sin ($ thi $), \cos ($ thi $), 0,0 ; 0,0,1,0 ; 0,0,0,1]$;

$>\operatorname{tam}=[1,0,0$, ai $; 0,1,0,0 ; 0,0,1,0 ; 0,0,0,1]$;

$>$ talm $=[1,0,0,0 ; 0, \cos ($ ali $),-\sin ($ ali) $), 0 ; 0, \sin ($ ali) $), \cos ($ ali $), 0 ; 0,0,0,1]$;

$>$ tim $=$ tbm*thm*tam8talm

Tim=

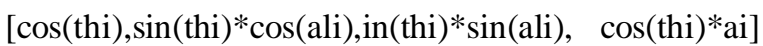

$[\sin ($ thi $), \cos ($ thi $) * \cos ($ ali $)-\cos ($ thi $) * \sin ($ ali $), \quad \sin ($ thi $) * a i]$ 
[0, $\sin ($ ali $)$, os(ali), bi]

$[0, \quad 0, \quad 0, \quad 1]$

Where command 'syms' are used to define the symbolic variables,'bi' 'thi,' 'ai,' and 'ali' for the DH parameter bi, $\theta$ i, ai, and $\alpha$ i respectively. Moreover,the notations,'tbm,' 'tthm,' and 'talm' are used to represent thye matrices $\mathrm{Tb}, \mathrm{T} \theta$, $\mathrm{Ta}$ and $\mathrm{T} \alpha$, given by eqs.(2),respectively.finally, the resultant matrix Ti, denoted as'tim' in the MATLAB environment, is evaluated above, which matches with eq.(2).[10]

\section{MOdeling OF Finger MeChanisM}

The convention outlined above does not result in a unique attachment of frames to links because alternative choices are available. For example: Joint axes i have two choices for direction to point zi-axis, one pointing upward (as in fig.2) and other pointing downward. To minimize such options and get a consistent set of frames, an algorithm 1.1 is presented below to assign frames to all links of a space mechanism.

Algorithm 1.1

This algorithm assigns frames and determines the DH-parameters for each link of an n-DOF manipulator .Both, the first link 0 and the last link n, are connected to only one other link and, thus, have more arbitrariness in frame assignment. For this reason, the first (frame $\{0\}$ )and the last (frame $\{\mathrm{n}\}$ ) frames are assigned after assigning frames to intermediate links, link 1 to link(n-1). The displacement of each joint-link is measured with respect to a frame; therefore the zero position of each link needs to be clearly defined. The zero position for a revolute joint is when the joint angle $\theta$ is zero, while for a prismatic joint it is when the joint displacement is minimal; it may or may not be zero. When all the joints are in zero position, the manipulator is said to be in home position. Thus, the home position of an n-DOF manipulator is the position where the $\mathrm{n} x 1$ vector of joint variables is equal to the zero vector, that is,$q i=0$ for $i=1,2, \ldots \ldots, n$.

Before assigning frames, the zero position of each joint, that is, the home position of the manipulator must be decided. The frames are then assigned imagining the manipulator in home position. Because of mechanical constraints, the range of joint motion possible is restricted and, in some cases, this may result in a home position that is unreachable. In such cases, the home position is redefined by changing the initial manipulator joint positions and/or frame assignments. The new home position can be obtained by adding a constant value to the joint angle in case of revolute joint and to the joint displacement in case of prismatic joint. This shifting of the home position is illustrated in design project, 6- DOF space mechanism.

The algorithm is divided into four parts. The first segment gives steps for labeling scheme and the second one describes the steps for frame assignment to intermediate links 1 to (n-1). The third and fourth segments give steps for frame $\{0\}$ and frame $\{n\}$ assignment, respectively.

Step 0 Identify and number the joints starting with base and ending with end-effector. Number the links from 0 to $n$ starting with immobile base as 0 and ending with last link as $\mathrm{n}$.

Step 1 Align axis zi with axis of joint ( $\mathrm{i}+1)$ for $\mathrm{i}=0,1, \ldots \ldots, \mathrm{n}-1$.

Assigning frames to intermediate links- link 1 to link (n-1) For each link repeat steps 2 and3.

Step 2 The xi-axis is fixed perpendicular to both zi-1 -and zi-axes and points away from zi-1. The origin of frame $\{i\}$ is located at the intersection of zi- and xi-axes. Three situations are possible:

Case (1) if zi-1-axes intersect; choose the origin at the point or their intersection. The xi-axis will be perpendicular to the plane containing zi-1-and zi-axes. This will give ai to be zero.

Case (2) If zi-1-and zi-axes are parallel or lie in parallel planes then their common normal is not uniquely defined. If joint $\mathrm{t}$ is revolute then $\mathrm{xi}$-axis is chosen along that common normal, which passes through origin of frame $\{i-1\}$. This will fix the origin and make di zero .if joint I is prismatic, xi-axis is located distal end of the link $\mathrm{i}$.

Case(3) if $\mathrm{Zi}-1$ and zi-axis coincide ,the origin lies on the common axis. if joint $\mathrm{I}$ is revolute, origin is located to coincide with origin of frame $\{\mathrm{i}-1\}$ and xi-axis coincide with xi-1 axis coincide with xi-1 axis to cause di to be zero. if joint $\mathrm{I}$ is prismatic, xi-axis to cause di to be 0 . If joint $\mathrm{I}$ is prismatic, xi-axis is chosen parallel to $\mathrm{xi}-1$ axis to make ai to be 0 . The origin is located at distal end of link $\mathrm{i}$.

Step3 the yi-axis has no choice and is fixed to right -handed orthonormal coordinate frame $\{\mathrm{i}\}$. Assigning frame to link 09 , the immobile base-frame $\{0\}$

Step4 The frame $\{0\}$ location is arbitrary. Its choice is made based on simplification of the model and some convenient reference in workspace. The $\mathrm{x} 0$-axis, which is perpendicular to $\mathrm{z} 0$-axis, is chosen to be parallel to $x i$-axis in the home position to make $\theta 1=0$. The origin of frame $\{0\}$ is located based on type of joint 1 . If joint 1 is revolute, the origin of frame $\{0\}$ can be chosen at a convenient reference such as, floor, work table, and so on, giving a constant value for parameter $\mathrm{d} 1$ zero.

If joint 1 is prismatic, parallel $\mathrm{x} 0$-and $\mathrm{x} 1$-axes will make $\theta 1$ to be zero and origin of frame $\{0\}$ is placed arbitrarily.

Step 5 The yo-axis completes the right-handed orthonormal coordinate frame $\{0\}$. 
Link $n$, the end-effector, frame assignment-frame $\{n\}$

Stape 6 The origin of frame $\{n\}$ is chosen at the tip of the manipulator, that is, a convenient point on the last link (the end -effecrtor). This point is called the tool point and the frame $\{n\}$ is the tool frame.

Step 7 The zn-axis is fixed along the direction of zn-1-axus and pointing away from the link $\mathrm{n}$. It is the direction of approach.

Step 8 If joint $\mathrm{n}$ is prismatic; take $\mathrm{xn}$ parallel to $\mathrm{xn}$-1-axis. If joint $\mathrm{n}$ is revolute, the choice of $\mathrm{xn}$ is similar to step 4, that is, $x n$ is perpendicular to both $\mathrm{zn}-1$-and zn-axes. Xn direction is the normal direction. The yn -axis is chosen to complete the right -handed orthonormal frame $\{\mathrm{n}\}$. The yn - axis is the "orientation" or "sliding" direction,[9]

\section{Result And Discussion}

Fig. 3 shows the model of the proposed Kinematic Model of Anthropomorphic robotics Finger mechanism. In Fig. 3, the fingers are assumed to be index, middle, ring and little fingers.

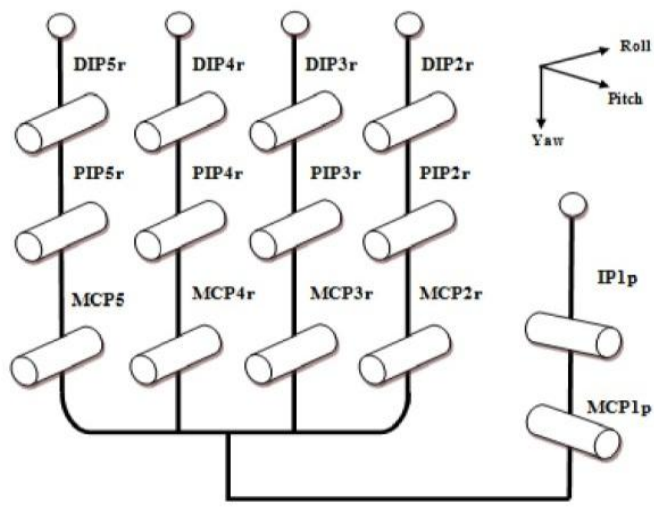

Fig.3: Model of a MFRH

The finger has 3 active joints. DIP joint has connection with PIP joint. The thumb is designed by having 2 active joints. The joint of each link of MFRH model is a frame to determine the kinematic derivation.

Fig. 4 shows that the finger has four frames with three joints. The first frame also known as the base frame is $\mathrm{x}_{0}, \mathrm{y}_{0}, \mathrm{z}_{0}$ and the subsequent frames are assigned as per the figure starting with $\mathrm{x}_{1}, \mathrm{y}_{1}, \mathrm{z}_{1}$ and ending with $\mathrm{x}_{4}, \mathrm{y}_{4}, \mathrm{z}_{4}$. The forward kinematic solution of a finger will be assigned using homogenous matrix.

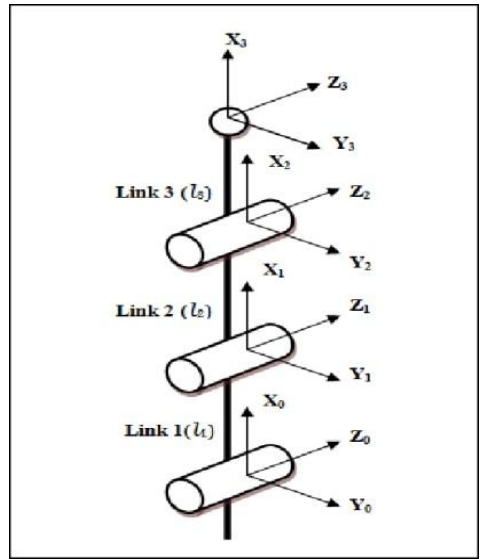

Fig.4:Single Finger Model

\section{Forward Kinematic}

Forward Kinematic is used to determine the position and orientation of MFRH to determine the position and orientation of the robot hand relative to the robot base coordinate system. The derivation of forward kinematic equation based on Table 1 
TABLE I

\begin{tabular}{ccccc}
\multicolumn{5}{c}{ TABLE I } \\
\hline \hline$i$ & $\theta_{i}$ & $d_{i}$ & $a_{i-1}$ & $\alpha_{i-1}$ \\
\hline 1 & $\theta_{1}$ & 0 & 0 & 0 \\
2 & $\theta_{2}$ & 0 & $l_{1}(\mathrm{MCP})$ & 0 \\
3 & $\theta_{3}$ & 0 & $l_{2}$ (PIP) & 0 \\
4 & 0 & 0 & $l_{3}$ (DIP) & 0 \\
\hline \hline
\end{tabular}

$$
\begin{gathered}
H_{i-0}^{i}=\left[\begin{array}{cccc}
c \theta_{i} & -s \theta_{i} & 0 & a_{i-1} \\
s \theta c \alpha_{i-1} & c \theta c \alpha_{i-1} & -s \alpha_{i-1} & -s \alpha_{i-1} d_{i} \\
c \theta s \alpha_{i-1} & c \theta s \alpha_{i-1} & c \alpha_{i-1} & c \alpha_{i-1} d_{i} \\
0 & 0 & 0 & 1
\end{array}\right] \\
H_{0}^{1}=\left[\begin{array}{cccc}
C_{1} & -S_{1} & 0 & 0 \\
S_{1} & C_{1} & 0 & 0 \\
0 & 0 & 1 & 0 \\
0 & 0 & 0 & 1
\end{array}\right] \\
H_{1}^{2}=\left[\begin{array}{cccc}
C_{2} & -S_{2} & 0 & l_{1} \\
S_{2} & C_{2} & 0 & 0 \\
0 & 0 & 1 & 0 \\
0 & 0 & 0 & 1
\end{array}\right] \\
H_{2}^{3}=\left[\begin{array}{cccc}
C_{3} & -S_{3} & 0 & l_{2} \\
S_{3} & C_{3} & 0 & 0 \\
0 & 0 & 1 & 0 \\
0 & 0 & 0 & 1
\end{array}\right]
\end{gathered}
$$

And

$$
H_{3}^{4}=\left[\begin{array}{llll}
1 & 0 & 0 & l_{3} \\
0 & 1 & 0 & 0 \\
0 & 0 & 1 & 0 \\
0 & 0 & 0 & 1
\end{array}\right]
$$

Hence, the forward kinematic for the fingers of robot hand are given by:

$$
H_{0}^{4}=\left[H_{0}^{1} H_{1}^{2} H_{2}^{3} H_{3}^{4}\right]
$$

We assume that,

$$
\begin{aligned}
& \cos \theta_{1}=C_{1}, \cos \theta_{2}=C_{2}, \cos \theta_{3}=C_{3} \text { and } \\
& \sin \theta_{1}=S_{1}, \sin \theta_{2}=S_{2}, \sin \theta_{3}=S_{3}
\end{aligned}
$$

First, find $H_{0}^{2}=H_{0}^{1} H_{1}^{2}$ 


$$
\begin{aligned}
& =\left[\begin{array}{cccc}
C_{1} & -S_{1} & 0 & 0 \\
S_{1} & C_{1} & 0 & 0 \\
0 & 0 & 1 & 0 \\
0 & 0 & 0 & 1
\end{array}\right]\left[\begin{array}{cccc}
C_{2} & -S_{2} & 0 & l_{1} \\
S_{2} & C_{2} & 0 & 0 \\
0 & 0 & 1 & 0 \\
0 & 0 & 0 & 1
\end{array}\right] \\
& =\left[\begin{array}{cccc}
C_{1} C_{2}-S_{1} S_{2} & -C_{1} S_{2}-S_{1} C_{2} & 0 & l_{1} C_{1} \\
S_{1} C_{2}+C_{1} S_{2} & -S_{1} S_{2}+C_{1} C_{2} & 0 & l_{1} S_{1} \\
0 & 0 & 1 & 0 \\
0 & 0 & 0 & 1
\end{array}\right]
\end{aligned}
$$

where,

$$
\begin{aligned}
& \cos \theta_{1} \cos \theta_{2}-\sin \theta_{1} \sin \theta_{2}=\cos \left(\theta_{1}+\theta_{2}\right)=C_{12} \\
& \sin \theta_{1} \cos \theta_{2}+\cos \theta_{1} \sin \theta_{2}=\sin \left(\theta_{1}+\theta_{2}\right)=S_{12}
\end{aligned}
$$

\section{Hence,}

$$
H_{0}^{2}=\left[\begin{array}{cccc}
C_{12} & -S_{12} & 0 & l_{1} C_{1} \\
S_{12} & C_{12} & 0 & l_{1} S_{1} \\
0 & 0 & 1 & 0 \\
0 & 0 & 0 & 1
\end{array}\right]
$$

Then, find $H_{0}^{3}=H_{0}^{1} H_{1}^{2} H_{2}^{3}$

$$
\begin{aligned}
& H_{0}^{2}=\left[\begin{array}{cccc}
C_{12} & -S_{12} & 0 & l_{1} C_{1} \\
S_{12} & C_{12} & 0 & l_{1} S_{1} \\
0 & 0 & 1 & 0 \\
0 & 0 & 0 & 1
\end{array}\right]\left[\begin{array}{cccc}
C_{3} & -S_{3} & 0 & l_{2} \\
S_{3} & C_{3} & 0 & 0 \\
0 & 0 & 1 & 0 \\
0 & 0 & 0 & 1
\end{array}\right] \\
& =\left[\begin{array}{cccc}
C_{12} C_{3}-S_{12} S_{3} & -C_{12} S_{3}-S_{12} C_{3} & 0 & l_{1} C_{1}+l_{2} C_{12} \\
S_{12} C_{3}+C_{12} S_{3} & -S_{12} S_{3}+C_{12} C_{3} & 0 & l_{1} S_{1}+l_{2} S_{12} \\
0 & 0 & 1 & 0 \\
0 & 0 & 0 & 1
\end{array}\right] \\
& \text { where, } \\
& \cos \theta_{12} \cos \theta_{3}-\sin \theta_{12} \sin \theta_{3}=\cos \left(\theta_{1}+\theta_{2}+\theta_{3}\right)=C_{123} \\
& \sin \theta_{12} \cos \theta_{3}+\cos \theta_{12} \sin \theta_{3}=\sin \left(\theta_{1}+\theta_{2}+\theta_{3}\right)=S_{123}
\end{aligned}
$$

Insert eq. (1) \& (2) in (3);

$$
\begin{aligned}
& =\cos \theta_{1} \cos \theta_{2} \cos \theta_{3}-\sin \theta_{1} \sin \theta_{2} \sin \theta_{3} \\
& =\left(\cos \theta_{1} \cos \theta_{2}\right) \cos \theta_{3}-\left(\sin \theta_{1} \sin \theta_{2}\right) \sin \theta_{3} \\
& =\cos \theta_{12} \cos \theta_{3}-\sin \theta_{12} \sin \theta_{3} \\
& =\cos \left(\theta_{1}+\theta_{2}+\theta_{3}\right)=C_{123}
\end{aligned}
$$




$$
\begin{array}{ll} 
& =\sin \theta_{1} \sin \theta_{2} \cos \theta_{3}+\cos \theta_{1} \cos \theta_{2} \sin \theta_{3} \\
& =\left(\sin \theta_{1} \sin \theta_{2}\right) \cos \theta_{3}+\left(\cos \theta_{1} \cos \theta_{2}\right) \sin \theta_{3} \\
& =\sin \theta_{12} \cos \theta_{3}+\cos \theta_{12} \sin \theta_{3} \\
\text { Insert eq. (1) \& (2) in 4; } \quad=\sin \left(\theta_{1}+\theta_{2}+\theta_{3}\right)=S_{123}
\end{array}
$$

$$
H_{0}^{3}=\left[\begin{array}{cccc}
C_{123} & -S_{123} & 0 & l_{1} C_{1}+l_{2} C_{12} \\
S_{123} & C_{123} & 0 & l_{1} S_{1}+l_{2} S_{12} \\
0 & 0 & 1 & 0 \\
0 & 0 & 0 & 1
\end{array}\right]
$$

Finally, the derivation of forward kinematic is

$$
H_{0}^{4}=\left[H_{0}^{1} H_{1}^{2} H_{2}^{3} H_{3}^{4}\right]
$$

$$
\begin{aligned}
H_{0}^{4} & =\left[\begin{array}{cccc}
C_{123} & -S_{123} & 0 & l_{1} C_{1}+l_{2} C_{12} \\
S_{123} & C_{123} & 0 & l_{1} S_{1}+l_{2} S_{12} \\
0 & 0 & 1 & 0 \\
0 & 0 & 0 & 1
\end{array}\right]\left[\begin{array}{cccc}
1 & 0 & 0 & l_{3} \\
0 & 1 & 0 & 0 \\
0 & 0 & 1 & 0 \\
0 & 0 & 0 & 1
\end{array}\right] \\
& =\left[\begin{array}{cccc}
C_{123} & -S_{123} & 0 & l_{1} C_{1}+l_{2} C_{12}+l_{3} C_{123} \\
S_{123} & C_{123} & 0 & l_{1} S_{1}+l_{2} S_{12}+l_{2} S_{123} \\
0 & 0 & 1 & 0 \\
0 & 0 & 0 & 1
\end{array}\right]
\end{aligned}
$$

Thus, this completes the kinematic modeling of anthropomorphic robot Finger mechanisms. These equations are used in the simulation of the design of anthropomorphic robot Finger mechanisms. The results of the simulation shall be communicated in the next publication.

\section{Conclusion}

The kinematic modeling plays an important role in simulation of anthropomorphic robot Finger mechanisms. In this paper, the complete derivation of the kinematic modeling of anthropomorphic robot Finger mechanisms was carried out to enable subsequent simulation work. The results will be published in future. Other work such as development of control algorithm and development of anthropomorphic robot Finger mechanisms will be addressed in the next phase of study.

\section{References}

[1]. Denavit, j.,R.S. Hartenberg." A kinematic Notation for Lower -Pair Mechanisms Based on Matrices." ASME Journal of Applied Mechanics, June 1955, 215-221.

[2]. Paul Richard P., "Robot Manipulator, mathematics, Programming, and control," MIT Press, Cambridge ,Mass., 1981.

[3]. Craig, John J., Introduction to Robotics: Mechanics and Contorl, 2d Edition, Addison Wesley, 1989.

[4]. Shahinpoor, Mohsen, A robot Engineering Textbook, Harper \&Row, Publishers, 1987

[5]. Koren, Yoram, Robotics for Engineers, McGraw-Hill, 1985.

[6]. Fu, K.S., R.C. Gonzalez and C.S.G.Lee, Robotics: Control, Sensing, Vision, and Inteliangence, McGraw-Hill, 1987

[7]. Neil A. Davidoff, Andris Freivalds (August4, 1993). "A graphic model of the human hand using CATIA". Department of Industrial and Management Systems Engineering, Pennsylvania State University, University Park, PA 16802, USA

[8]. Jung Won Kang, Hyun Seok Hong, Bong Sung Kim, Myung Jin Chung, "Work Assistive Mobile Robots Assisting the Disabled in a Real Manufacturing Environment”, International Journal of Assistive Robotics and Mechtronics, Volume 8 Number 3, September 2007

[9]. S.C. Jacobsen, E.K. Inversen, D.F. Knutti, R. T. Johnson and K.B. Biggers, "Design of the Utah/MIT Dexterous Hand, Proceeding s of the 1986 IEEE International Conference on Robotics and Automation, pp 1520-1532, 1986

[10]. Matlab, 2002,MATLAB- The language of computing, version 6.5, Release 13. 